\title{
A randomised clinical trial of oral steroids in the treatment of carpal tunnel syndrome: a long term follow up
}

\author{
M-H Chang, L-P Ger, P F Hsieh, S-Y Huang
}

See end of article for authors' affiliations ......................

Correspondence to: Dr M-H Chang, Section of Neurology, Taichung Veterans General Hospital, 160 Chung Kang Road, $\mathrm{Sec} 3$, Taichung, Taiwan 40705;

cmh50@ms10.hinet.net

Received

15 January 2002

In revised form

15 July 2002

Accepted

5 September 2002
Objectives: To determine the efficacy of a two week and a four week course of oral steroids in the conservative treatment of carpal tunnel syndrome.

Methods: 109 patients with carpal tunnel syndrome were randomly divided into two treatment groups: (1) two weeks of prednisolone $20 \mathrm{mg}$ daily followed by two weeks of prednisolone $10 \mathrm{mg}$ daily $(\mathrm{n}=53)$; (2) two weeks of prednisolone $20 \mathrm{mg}$ daily and two weeks of placebo $(\mathrm{n}=56)$. A symptom questionnaire was used to rate the five major symptoms of carpal tunnel syndrome (numbness, pain, weakness/clumsiness, tingling, and nocturnal awakening) on a scale of 0 (nil) to 10 (severe); the resulting global symptom score was used to evaluate the efficacy of treatment. Assessments were made at baseline and at one, three, six, nine, and 12 months. Electrodiagnosis was repeated at the end of the study to validate improvement.

Results: In an intention to treat analysis at the end of the study, improvement in the four week treatment group was achieved in $66.0 \%$ of the patients after one month and in $49.0 \%$ at the end of the study; in the two week treatment group, the respective values were $48.2 \%$ and $35.7 \%$. In the four week treatment group, $51 \%$ were considered treatment failures (including those lost to follow up, receiving surgery, or with mild or no improvement), compared with $64.3 \%$ for the two week group. Though the percentage improvement was higher in the four week group, the difference did not reach a statistical significance. Persistence of improvement was $74.2 \%$ in the four week group $v 74.1 \%$ in the two week group, suggesting no difference in the long term effect. Efficacy analysis showed no significant difference in global symptom score reduction between the two groups. Follow up electrodiagnosis showed significant improvement in all measured variables except for the amplitude of compound muscle action potentials.

Conclusions: Short term low dose oral steroid are effective treatment for carpal tunnel syndrome. The dose of steroids and the duration treatment are not key determinants of efficacy.
$\mathrm{T}$ he symptoms of carpal tunnel syndrome are numbness or tingling pain in the wrist, hands, and fingers, often occurring during the night and waking the patient from sleep. Relief is usually obtained by shaking or rubbing the hands. ${ }^{1-3}$ The best way to confirm the diagnosis is to carry out a median nerve conduction study across the transverse carpal tunnel ligament. Characteristic abnormalities are a focal reduction in conduction velocity over the distal motor or sensory fibres, with or without reduced amplitudes of the compound action potentials. The clinical manifestations ${ }^{1-3}$ and electrodiagnostic criteria of carpal tunnel syndrome have been well documented..$^{4-6}$ However, conservative treatment for mild to moderate expressions of the syndrome varies. Non-operative treatment usually involves the use of local steroid injections and short term immobilisation of the wrist in a splint. Many studies have addressed the effectiveness of this type of conservative treatment and have found that recurrence is common. ${ }^{7-10}$ Drug treatment is often used in patients with carpal tunnel syndrome. ${ }^{12}{ }^{10-13}$ In a previous study, we compared the effectiveness of non-steroidal anti-inflammatory drugs, diuretics, and oral steroids in the treatment of carpal tunnel syndrome over a four week period. ${ }^{14}$ The results showed that steroids were more beneficial than the other agents. However, some uncertainties remained. For example, we did not know how effective steroid treatment was for long term symptom relief, or whether a short course or a longer course of treatment was preferable. To address these issues, we conducted a one year prospective randomised study.

\section{METHODS}

Patients and electrophysiological assessment

The patients enrolled in the study had clinical symptoms and signs of carpal tunnel syndrome confirmed by standard electrodiagnostic tests. Testing the radial and ulnar nerves showed that they had no abnormalities. Motor and sensory nerve conduction studies were done using standard techniques of supramaximal percutaneous stimulation and surface electrode recording. The nerves sampled were the median, ulnar, and radial nerves. Amplitude and conduction velocity of compound muscle action potentials (CMAP) and sensory nerve action potentials (SNAP) were measured using to the method described by Delisa et al..$^{15}$ The electromyographic recordings (Viking IV, Nicolet, Madison, Wisconsin, USA) of motor conduction velocity were made with the filter bandpass at $2 \mathrm{~Hz}$ to $10 \mathrm{kHz}$, a sweep speed of $2 \mathrm{~ms} / \mathrm{cm}$, and the amplifier gain adjusted for viewing the CMAP. For measurement of SNAP, the instrument settings were: filters, $20 \mathrm{~Hz}$ to $10 \mathrm{kHz}$; sweep, 2 $\mathrm{ms} / \mathrm{cm}$; gain, $10-20 \mu \mathrm{V} / \mathrm{cm}$.

The electrophysiological criteria for the diagnosis of carpal tunnel syndrome were median sensory distal latency (SDL) more than $3.1 \mathrm{~ms}$ or a mixed or sensory median nerve wristpalm conduction time $(\mathrm{W}-\mathrm{P})$ greater than $2.0 \mathrm{~ms}$ at a distance of $8 \mathrm{~cm}$, and motor distal latency (MDL) more than $4.7 \mathrm{~ms}$ (mean value $+3 \mathrm{SD}) .^{14} 16$

Exclusion criteria were as follows:

- symptoms occurring less than three months before the study (to exclude patients who might have spontaneous resolution of symptoms); 
- the presence of either fibrillation potentials or reinnervation on needle electromyography (EMG) in the abductor pollicis brevis muscle (to ensure the inclusion of only mildly or moderately affected individuals); "mild" describes patients with decreased conduction velocity over the palmwrist segment and delayed SDL, with normal median SNAP amplitude and CMAP amplitude of the abductor pollicis brevis; "moderate" describes patients with abnormally delayed MDL and SDL with either decreased median SNAP amplitude or decreased CMAP amplitude of the abductor pollicis brevis;

- clinical or electrophysiological evidence of accompanying conditions that could mimic carpal tunnel syndrome or interfere with its evaluation such as cervical radiculopathy, proximal median neuropathy, or significant polyneuropathy;

- evidence of obvious underlying causes of carpal tunnel syndrome such as hypothyroidism, diabetes mellitus, arthritis of the wrist, pregnancy, or the use of vibrating machinery;

- cognitive impairment interfering with the subject's ability to follow instructions and describe symptoms;

- recent peptic ulcer or a history of steroid intolerance.

\section{Treatment protocol}

The study protocol was approved by the institutional review board of our hospital. If patients fulfilled the criteria and gave consent, they were enrolled in the study. At their first visit we assessed their medical and neurological history in a standardised way, carried out biochemical and endocrine screening (including measurement of fasting blood sugar, renal function, rheumatoid factor, thyroid stimulating hormone, triiodothyronine, and thyroxine), and carried out a detailed physical and neurological examination, nerve conduction studies, and needle EMG. Before drug treatment was begun, the patients were observed and followed up for one month. If improvement occurred during that time, they were excluded from the study.

After enrolment, the patients were randomised into two treatment groups: ( 1) a group receiving $20 \mathrm{mg}$ of prednisolone daily for two weeks, followed by $10 \mathrm{mg}$ daily for two weeks; (2) a group receiving $20 \mathrm{mg}$ of prednisolone daily for two weeks, followed by placebo for two weeks.

\section{Measurement of response}

Assessments included a symptom questionnaire modified from that used by Herskovitz et al and You et al, ${ }^{13}{ }^{17}$ and by us in our previous study, ${ }^{14}$ which rates symptoms from 0 (no symptoms) to 10 (very severe symptoms) in each of five categories: pain, numbness, tingling, weakness/clumsiness, and nocturnal awakening.

The scores for pain, numbness, and tingling were made up of subscores for magnitude, frequency, and duration in one day, as follows: magnitude (total possible score 4): none, 0; mild, 1; moderate, 2; severe, 3; very severe, 4; frequency (total possible score 3 ): never, 0 ; once or twice, 1 ; three to five times, 2 ; more than five times, 3 ; duration (total possible score 3 ): none, 0 ; less than 10 minutes, 1 ; 10 to 60 minutes, 2; more than 60 minutes, 3 .

The score for nocturnal awakening was determined by times wakened in one week: never, 0 ; once or twice, 2 ; three or four times, 4; five to seven times, 6; eight to 10 times, 8; more than 10 times, 10 .

The score for weakness was assessed according to the severity of the weakness: none, 0 ; mild, 2; moderate, 3; severe, 4; very severe, 5 ; and for clumsiness by difficulty in manipulating small objects: none, 0; mild, 2; moderate, 3; severe, 4; very severe, 5 .

The total of the scores for the five categories formed the global symptom score. Each score was determined by direct
Table 1 Summary of demographic findings and adverse effects

\begin{tabular}{lll}
\hline & $\begin{array}{l}4 \text { Weeks } \\
\text { treatment }\end{array}$ & $\begin{array}{l}2 \text { Weeks } \\
\text { treatment }\end{array}$ \\
\hline $\mathrm{n}$ & 53 & 56 \\
Age (years) & $46.2(5.4)$ & $45.9(5.1)$ \\
Sex (female/male) & $40 / 13$ & $41 / 15$ \\
Symptoms & & \\
$\quad$ Duration (months) & $6.2(2.8)$ & $6.6(3.2)$ \\
Numbness & 53 & 56 \\
Tingling & 36 & 34 \\
Pain & 29 & 28 \\
Weakness & 25 & 21 \\
Nocturnal waking & 47 & 43 \\
& & \\
Median nerve conduction study & \\
MDL (ms) & $5.8(1.1)$ & $5.9(1.1)$ \\
CMAP (mv) & $10.6(2.6)$ & $9.2(1.7)$ \\
SNCV (m/s) & $37.9(6.6)$ & $37.9(5.9)$ \\
W-P (ms) & $2.8(0.7)$ & $2.8(0.5)$ \\
SNAP ( $\mu V$ V) & $20.8(15.0)$ & $17.8(9.6)$ \\
Adverse effect & & \\
Nausea & 3 & 4 \\
Epigastralgia & 5 & 3 \\
Raised blood sugar & 2 & 3 \\
\hline
\end{tabular}

Values are $\mathrm{n}$ or mean (SD)

CMAP, compound muscle action potential; MDL, motor distal latency;

SNAP, sensory nerve action potential; $\mathrm{SNCV}$, sensory nerve

conduction velocity; W-P, wrist-palm conduction time.

questioning of the patient and was based on the patient's subjective answers. The maximum score was 50 (the most severe symptoms) and the minimum score was 0 (absence of any symptoms).

Before the end of the study, some of the patients received surgical ligament release, sought alternative treatments, or were lost during follow up. Thus an intention to treat analysis was created and all of the patients, including those with complete and incomplete follow up, were included in the analysis. For individual response to steroids, a reduction in the global symptom score by more than $50 \%$ placed a patient in the improved group (a reduction of more than $75 \%$ was classed as marked improvement and $50-75 \%$ as moderate improvement). A reduction in the global symptom score of $50 \%$ or less placed a patient in the failed group (a reduction of $25-50 \%$ was classed as only minor improvement, and less than $25 \%$ as no improvement). We included patients who underwent surgery or who were lost during follow up in the "failed" group. The efficacy analysis only involved those patients who actually finished the study.

The randomisation list was developed and kept by a person not involved in the care or evaluation of the patients or in the data analysis. Neither the patients nor the physicians were aware of the treatment allocation. Access to the treatment was restricted. Furthermore, to ensure consistency, the evaluating physician was the same person on each occasion for each patient. Follow up assessments-identical to the baseline procedure-were done at one, three, six, nine, and 12 months. At the end of the assessment, physical and neurological examinations were repeated, along with the same biochemical and endocrine examinations as at baseline. Because some subjects in the failed group were lost to follow up, received surgery, or refused to have any more electrodiagnostic tests, we decided not to repeat the electrodiagnostic evaluation in the whole population; however, in order to obtain objective evidence of improvement, repeat nerve conduction studies were undertaken in those patients who showed symptomatic improvement.

We recorded adverse side effects such as nausea, epigastric pain, tarry stools, leg oedema, or cushingoid appearance, along 
Table 2 Summarised results of intention to treat analysis

\begin{tabular}{lllllll}
\hline \multicolumn{5}{c}{ Four week treatment $(\mathrm{n}=53) /$ two week treatment $(\mathrm{n}=56)$} \\
\cline { 2 - 6 } & Result & Month 1 & Month 3 & Month 6 & Month 9 & Month 12 \\
\hline Improvement (\%) & Overall & $66.0 / 48.2(35 / 27)$ & $64.1 / 44.6(34 / 25)$ & $52.8 / 37.5(28 / 21)$ & $50.9 / 37.5(27 / 21)$ & $49.0 / 35.7(26 / 20)$ \\
$(4 \mathrm{wk} / 2 \mathrm{wk})$ & Marked effect & $37.7 / 33.9(20 / 19)$ & $37.7 / 35.7(20 / 20)$ & $45.3 / 26.8(24 / 15)$ & $39.6 / 26.8(21 / 15)$ & $35.8 / 25(19 / 14)$ \\
& Moderate effect & $28.3 / 14.3(15 / 8)$ & $26.4 / 8.9(14 / 5)$ & $7.5 / 10.7(4 / 6)$ & $11.3 / 10.7(6 / 6)$ & $13.2 / 10.7(7 / 6)$ \\
Failure (\%) & Overall & $34.0 / 51.8(18 / 29)$ & $35.9 / 55.4(19 / 31)$ & $47.2 / 62.5(25 / 35)$ & $49.1 / 62.5(26 / 35)$ & $51.0 / 64.3(27 / 36)$ \\
$(4 \mathrm{wk} / 2 \mathrm{wk})$ & Mild effect & $11.3 / 17.8(6 / 10)$ & $11.3 / 10.7(6 / 6)$ & $11.3 / 8.9(6 / 5)$ & $5.7 / 3.6(3 / 2)$ & $3.8 / 3.6(2 / 2)$ \\
& No effect & $18.9 / 23.2(10 / 13)$ & $15.1 / 17.9(8 / 10)$ & $20.8 / 19.6(11 / 11)$ & $22.6 / 23.2(12 / 13)$ & $26.4 / 25.1(14 / 14)$ \\
& Loss of follow up & $3.8 / 7.1(2 / 4)$ & $7.6 / 17.8(4 / 10)$ & $9.5 / 19.6(5 / 1)$ & $11.3 / 19.6(6 / 11)$ & $11.3 / 19.6(6 / 11)$ \\
& Surgery & $0 / 3.6(0 / 2)$ & $1.9 / 8.9(1 / 5)$ & $5.7 / 14.2(4 / 8)$ & $9.5 / 16(5 / 9)$ & $9.5 / 16(5 / 9)$ \\
\hline \multirow{2}{*}{ Figures in brackets are actual patient numbers. } & & & & \\
\hline
\end{tabular}

Table 3 Summarised results of efficacy analysis

\begin{tabular}{|c|c|c|c|c|c|c|}
\hline & \multicolumn{6}{|c|}{ Four week treatment $(n=42) /$ two week treatment $(n=36)$} \\
\hline & Baseline & Month 1 & Month 3 & Month 6 & Month 9 & Month 12 \\
\hline $\begin{array}{l}\text { Global symptom } \\
\text { scores (GSS) }\end{array}$ & $\begin{array}{l}17.88(6.06) / \\
17.24(4.95)\end{array}$ & $\begin{array}{l}8.1(6.8)^{*} / \\
9.54(6.56)^{*}\end{array}$ & $\begin{array}{l}8.08(7.72)^{*} / \\
9.1(7.95)^{*}\end{array}$ & $\begin{array}{l}9.02(9.19)^{*} / \\
9.65(8.27)^{*}\end{array}$ & $\begin{array}{l}9.4(10.18)^{*} / \\
10.25(9.21)^{*}\end{array}$ & $\begin{array}{l}10.25(10.6)^{*} / \\
10.61(9.15)^{*}\end{array}$ \\
\hline
\end{tabular}

with blood pressure and blood sugar measurements. No alterations in daily activites or additional treatments (such as splinting or and local injections) were permitted during the study.

\section{Statistical analysis}

We carried out two analyses: an intention to treat analysis for all enrolled patients, and an efficacy analysis for those who completed the one year follow up. To compare the improvement and failure in the two week and four week treatment groups, we used $\chi^{2}$ tests for the intention to treat analysis. In the efficacy analysis, a paired $t$ test was used to compare baseline and the effects of oral steroid in each follow up period. To compare the efficacy between the two week and four week group, we used a two sample $t$ test. A probability (p) value of less than 0.05 was considered significant. For the electrodiagnostic improvement, a paired $t$ test was used to determine the significance between baseline and the status at the end of the study.

\section{RESULTS}

\section{Enrolment of patients and baseline characteristics}

From January 1998 to December 2000, 138 patients were diagnosed as having carpal tunnel syndrome by clinical and electrodiagnostic criteria, fulfilling the neurophysiological classification of moderate severity. ${ }^{18}$ In 15 patients there was evidence of denervation of the adductor pollicis brevis muscle and these had surgical treatment. Eleven patients refused to be enrolled in the study and in three the symptoms improved during the one month observation period. In all, therefore, 109 patients agreed to participate in the study and were randomly allocated to the two week or four week treatment group.

The baseline characteristics of the two groups were similar (table 1). Of the 109 patients, $11(20.8 \%)$ assigned to the four week treatment $(\mathrm{n}=53)$ and $20(35.6 \%)$ assigned to the two week treatment $(n=56)$ did not finish the study. Fourteen patients (five in the four week group, nine in the two week group) received surgery before the end of the study, and 17 patients (six in the four week group, 11 in the two week group) were lost to or refused further follow up. Thus 78 patients (42 in the four week treatment group and 36 in the two week treatment group) were available for the efficacy analysis.
Table 4 Improvement in electrodiagnostic measurements in patients with carpal tunnel syndrome who had symptom relief

\begin{tabular}{lll}
\hline $\begin{array}{l}\text { Electrodiagnostic variable, } \\
\text { with normal result }\end{array}$ & $\begin{array}{l}\text { Before steroid } \\
\text { treatment }(\mathrm{n}=46)\end{array}$ & $\begin{array}{l}\text { One year later } \\
(\mathrm{n}=46)\end{array}$ \\
\hline MDL $(\mathrm{ms}),<4.7$ & $5.25(0.66)$ & $4.92(0.91)^{*}$ \\
CMAP $(\mathrm{mv}),>6.5$ & $10.77(2.21)$ & $10.6(1.92)$ \\
SNCV $(\mathrm{m} / \mathrm{s}),>45$ & $41.44(4.45)$ & $45.84(4.7)^{*}$ \\
W-P $(\mathrm{ms}),<2.0$ & $2.49(0.37)$ & $2.20(0.36)^{*}$ \\
SNAP $(\mu v),>15$ & $24.78(12.77)$ & $26.08(11.37)^{*}$ \\
\hline
\end{tabular}

Values are mean (SD)

${ }^{*} \mathrm{p}<0.05$.

CMAP, compound muscle action potential; MDL, median distal motor latency; SNAP, median sensory nerve action potential; SNCV, median sensory nerve conduction velocity; W-P, wrist-palm conduction time.

\section{Outcome of treatment}

Table 2 shows the outcome of all the patients randomly assigned to treatment, as determined by the intention to treat analysis. At the end of the study, there was a greater percentage of improvement in the four week group than in the two week group ( $49 \%$ v 35.7\%), though statistical significance was not achieved. There were also fewer patients with failed treatment in the four week group. In the four week group, $11.3 \%$ were lost to follow up or refused to continue, $9.5 \%$ had surgery, and $30.2 \%$ had either no improvement $(26.4 \%)$ or only minor improvement $(3.8 \%)$. In comparison, the rate of failed treatment in the two week group was $19.6 \%$ lost to follow up, $16 \%$ receiving surgery, and $28.7 \%$ with either no improvement $(25.1 \%)$ or only minor improvement $(3.6 \%)$. The cumulative failure rate was $51 \%$ in the four week treatment group and $64.3 \%$ in the two week group (NS). If a patient had a good response initially, we could predict a good response in the long term. Persistence of positive response was $74.2 \%(26 / 35)$ in the four week treatment group and $74.1 \%(20 / 27)$ in the two week group. There was no significant difference in response persistence between the two groups.

Table 3 shows the change in symptom scores for the 78 patients who were available for the efficacy analysis. The global symptom score were significantly decreased in the first, 
third, sixth, ninth, and 12th month as compared with the baseline scores in both treatment groups. There was no significant difference between the four week group and the two week group.

At the end of the study, 46 patients showed marked or moderate improvement (26 in the four week group and 20 in the two week group). Nerve conduction studies were repeated in these (table 4). There was significantly decreased MDL and W-P, and increased SNCV and SNAP amplitudes, but changes in CMAP amplitudes did not reach significance. The W-P in nine patients worsened, six showed no change, and 31 showed a decrease. Twelve patients had normal W-P in the retested nerve conduction study. Repeated biochemistry and endocrine screening tests showed that two patients in the four week group and three in the two week group had a raised blood sugar.

\section{DISCUSSION}

Steroids are effective at reducing swelling on account of their anti-inflammatory action. It is thus reasonable to use oral steroids in the treatment of carpal tunnel syndrome. ${ }^{12-14} 1920$ Our pilot study ${ }^{14}$ and three placebo control studies ${ }^{13} 1920$ showed that in the short term a course of low dose steroids can be of great benefit to patients with mild or moderate carpal tunnel syndrome, though the duration of the treatment course and the doses used were different in each of those studies. However, they only included a short period of follow up, so the long term efficacy of oral steroid treatment remained uncertain. It was for that reason that we undertook the present study, with a much longer follow up of one year. Our results show that a short low dose course of oral steroids is useful and effective in providing long term symptom relief in carpal tunnel syndrome. We therefore conclude that before advising surgery, conservative treatment-including splinting and systemic or local steroid use-should be given to all patients with carpal tunnel syndrome who do not have obvious motor and sensory impairment.

The natural history of the carpal tunnel syndrome was not well known until a recent study by an Italian group. ${ }^{21}$ This group found that between $20 \%$ and $30 \%$ of patients with carpal tunnel syndrome and abnormally delayed MDL and SDL (who thus belonged to the moderate severity group according to the neurophysiological classification ${ }^{18}$ ) had $20-30 \%$ spontaneous improvement in their symptomatic and functional scores. In the remainder the symptoms remained unchanged or worsened. It was clearly shown that spontaneous improvement in the moderate severity group was less common than originally thought. ${ }^{22}$ In the design of our present study, we were aware that a prospective, randomised, double blind, placebo controlled trial was the ideal method of evaluating the efficacy of treatment; however, it may be ethically difficult to persuade patients to continue follow up for one year if they have served as part of a placebo group. Thus we hoped to use the results of the Italian carpal tunnel syndrome study group $^{21}$ as a reference. According to the neurophysiological classification, all 109 of our patients had carpal tunnel syndrome of moderate severity. The patient oriented symptom scores showed a more than $50 \%$ decrease in $49 \%$ of the patients in the four week treatment group and in $35.7 \%$ in the two week group. The improvement rate and degree were thus more marked in our study than in the Italian study, suggesting that low dose, short term steroid treatment is effective in treating this condition when it is of moderate severity. In one previous study, nearly a quarter of the patients showed resolution of their symptoms within one month of initial assessment. $^{10}$ To minimise this confounding effect, we employed a one month initial observation period before starting steroid treatment. Any patients whose symptoms resolved spontaneously during that observation period were excluded from the study. Before entering the study, only three patients had marked improvement in their symptoms during the observation period and those were excluded. Though no placebo or other treatments were used for a comparison group in our study, a placebo effect or spontaneous resolution were unlikely outcomes, given our strict study design. Furthermore, we used electrodiagnostic measurements for objective assessment. There was marked improvement in the electrodiagnostic measurements in the patients who had marked or moderate improvement in the carpal tunnel symptoms. Thus, even though our study did not have a placebo controlled design, we feel we can exclude a placebo effect as the cause of the patients' improvement. Nonetheless, to investigate further the issue of spontaneous improvement and possible placebo effects, a randomised, double blind, placebo controlled study is now under way.

Oral steroids are rarely used to treat carpal tunnel syndrome, particularly in the USA. ${ }^{10}$ Herskovitz et al first described the effectiveness of low dose short term steroid treatment in the management of six patients with carpal tunnel syndrome. ${ }^{13}$ They prescribed $20 \mathrm{mg}$ prednisone daily for the first week, followed by a second week of $10 \mathrm{mg}$ daily; however, in the patients who improved, the effect seemed to be rather short lived. We therefore enrolled more patients and changed the dose to two weeks of $20 \mathrm{mg}$ prednisolone daily and then two weeks of $10 \mathrm{mg}$ daily. ${ }^{14}$ With this course, the period of efficacy was longer than in Herskovitz's study. In two recent small placebo controlled studies, ${ }^{19} 20$ a 10 day course of $25 \mathrm{mg}$ prednisolone daily was prescribed and the results showed significant improvement in the global symptom score in the steroid group. However, the most appropriate treatment period and dose remain unknown.

In order to avoid side effects, a low dose, short term course of oral steroids was selected for the present study. Our results show that four weeks of treatment did not result in significantly greater improvement that two weeks of treatment. Furthermore, the long term improvement rates were similar in the four week and the two week treatment groups $(74.3 \% \vee 74.1 \%)$. This suggests that the dose and duration of oral steroids used for treating carpal tunnel syndrome are not key determinants of efficacy.

\section{ACKNOWLEDGEMENTS}

This study was supported by grants VGHKS 88-08, NSC 90-2314-B075A-005, and TCVGH 903405N, 913406D, and 923402C.

\section{Authors' affiliations}

M-H Chang, P F Hsieh, S-Y Huang, Section of Neurology, Taichung Veterans General Hospital and Department of Neurology, National Yang-Ming University, Taipei and Chung-Shan Medical University, Taichung, Taiwan

L-P Ger, Department of Medical Research and Education, Kaohsiung Veterans General Hospital, Taiwan

Competing interests: none declared

\section{REFERENCES}

1 Spinner RJ, Bachman JW, Amadio PC. The many faces of carpal tunnel syndrome. Mayo Clin Proc 1989:64:829-36.

2 Quality Standards Subcommittee, American Academy of Neurology. Practice parameter for carpal tunnel syndrome (summary statement). Neurology 1993;43:2406-9.

3 Katz RT. Carpal tunnel syndrome: a practical review. Am Fam Physician 1994:49:1371-9.

4 American Association of Electrodiagnostic Medicine Quality Assurance Committee. Literature review of the usefulness of nerve conduction studies and electromyography for the evaluation of patients with carpal tunnel syndrome. Muscle Nerve 1993;16:1392-414.

5 American Academy of Neurology, American Association of Electrodiagnostic Medicine, American Academy of Physical Medicine and Rehabilitation. Practice parameter for electrodiagnostic studies in carpal tunnel syndrome (summary statement). Neurology 1993;43:2404-5.

6 Stevens JC. AAEM minimonograph No 26. The electrodiagnosis of carpal tunnel syndrome. Muscle Nerve 1997;20:1477-86. 
7 Gelberman RH, Aronson D, Weisman MH. Carpal tunnel syndrome. Results of a prospective trial of steroid injection and splinting. J Bone Joint Surg Am 1980;62:1181-4.

8 Girlanda P, Dattola R, Venuto C, et al. Local steroid treatment in idiopathic carpal tunnel syndrome: short- and long-term efficacy. J Neurol 1993;240: 187-90.

9 Weiss AP, Sachar K, Gendreau M. Conservative management of carpal tunnel syndrome: a reexamination of steroid injection and splinting. J Hand Surg Am 1994;19:410-15.

10 DeStefano F, Nordstrom DL, Vierkant RA. Long-term symptom outcomes of carpal tunnel syndrome and its treatment. $J$ Hand Surg 1997;22A:200-10.

11 Ellis JM. Treatment of carpal tunnel syndrome with vitamin B6. South Med J 1987:80:882-4.

12 Duncan KH, Lewis RC, Foreman KA, et al. Treatment of carpal tunnel syndrome by members of the American Society for Surgery of the Hand: results of a questionnaire. J Hand Surg Am 1987;12:384-91.

13 Herskovitz S, Berger AR, Lipton RB. Low-dose, short-term oral prednisone in the treatment of carpal tunnel syndrome. Neurology 1995:45: 1923-5.

14 Chang MH, Chiang HT, Lee SSJ, et al. Oral drug of choice in carpal tunnel syndrome. Neurology 1998;51:390-3.
15 Delisa JA, Mackenzie K, Baran EM. Manual of nerve conduction velocity and somatosensory evoked potentials, 2nd ed. New York: Raven Press, 1987.

16 Chang MH, Liao KK, Chang SP, et al. Proximal slowing in carpal tunnel syndrome resulting from either conduction block or retrograde degeneration. J Neurol 1993;240:287-90.

17 You H, Simmons Z, Freivalds A, et al. Relationships between clinical symptom severity scales and nerve conduction measures in carpal tunnel syndrome. Muscle Nerve 1999;22:497-501.

18 Padua L, Lo Monaco M, Gregori B, et al. Neurophysiological classification and sensitivity in 500 carpal tunnel syndrome hands. Acta Neurol Scand 1997:96:211-17.

19 Wong SM, Hui ACF, Tang A, et al. Local vs systemic corticosteroids in the treatment of carpal tunnel syndrome. Neurology 2001;56:1565-7.

20 Hui ACF, Wong SM, Wong KS, et al. Oral steroid in the treatment of carpal tunnel syndrome. Ann Rheum Dis 2001;60:813-14.

21 Padua L, Padua R, Aprile I, et al. Multiperspective follow-up of untreated carpal tunnel syndrome. A multicenter study. Neurology 2001;56:1459-66.

22 Muhlau G, Both R, Kunath H. Carpal tunnel syndrome - course and prognosis. J Neurol 1984;231:83-6.

\section{The British Neuropsychiatry Association 2003 Annual Meeting}

The British Neuropsychiatry Association 2003 Annual Meeting will be held at the Institute of Child Health, central London on 13-14 February 2003. The meeting will cover the topics: "Recovering from head injury", "Medico-legal aspects of neuropsychiatry", and "The neuropsychiatry of love (A feast for Valentine's Day)". The meeting includes keynote addresses from prominent international and UK speakers, along with a session for members' contributions.

Additionally, on 12 February 2003, BNPA are holding a conference sponsored by the Institute of Social Psychiatry on "Stepping out after brain injury"; this will be linked to the BNPA conference session on "Recovering from head injury".

For further information please contact: Gwen Cutmore, BNPA Conference Secretary, Landbreach Boatyard, Chelmer Terrace, Maldon, Essex CM9 5HT (tel/fax: +44 (0) 1621 843 334; email: gwen.cutmore@lineone.net; website: www.bnpa.fsnet.co.uk).

For details of membership to the BNPA, open to medical practitioners in psychiatry, neurology, and related clinical neurosciences please contact: The Secretary, Professor A S David, Department of Psychological Medicine, Institute of Psychiatry, De Crespigny Park, Denmark Hill, London SE5 8AF, UK. 